\title{
Statistical Simulation of the Performance and Degradation of a PEMFC Membrane Electrode Assembly
}

\author{
David Harvey ${ }^{1}$, Alexander Bellemare-Davis ${ }^{1}$, Kunal Karan ${ }^{2}$, Barath Jayansankar ${ }^{2}$, Jon \\ Pharoah $^{2}$, Vesna Colbow ${ }^{1}$, Alan Young ${ }^{1}$, and Silvia Wessel ${ }^{1}$ \\ ${ }^{1}$ Ballard Power Systems, 9000 Glenlyon Parkway, Burnaby BC, Canada, V5J 5J8 \\ ${ }^{2}$ Queen's-RMC Fuel Cell Research Centre, 945 Princess Street, Kingston, Ontario, \\ Canada K7L 3N6
}

\begin{abstract}
A 1-D MEA Performance model was developed that considered transport of liquid water, agglomerate catalyst structure, and the statistical variation of the MEA characteristic parameters. The model was validated against a low surface area carbon supported catalyst across various platinum loadings and operational conditions. The statistical variation was found to play a significant role in creating noise in the validation data and that there was a coupling effect between movement in material properties with liquid water transport. Further, in studying the low platinum loaded catalyst layers it was found that liquid water played a significant role in the increasing the overall transport losses. The model was then further applied to study platinum dissolution via potential cycling accelerated stress tests, in which the platinum was found to dissolve nearest the membrane effectively resulting in reaction distribution shifts within the layer.
\end{abstract}

\section{Introduction}

Improvements in the beginning of life (BOL) performance and load-cycle durability of the membrane electrode assembly (MEA) continue to be key areas of focus for PEM fuel cells (1), for both research and engineering development. Specifically, reductions in the platinum loading, while maintaining performance and durability are key aspects to meeting the cost targets necessary for commercialization. However, meaningful reductions in platinum loading have been observed to result in unexpected additional performance losses, increased sensitivity to operational conditions, and lower tolerance to voltage/load cycling resulting in decreased durability (2 - 4).

In order to facilitate the optimization of the BOL performance and mitigation of the performance loss over time due to voltage cycling; a statistically-based transient MEA performance and durability model has been developed. The model includes liquid water transport, a composition-based approach for both the transport and electrochemical parameters, and platinum dissolution/transport/plating. The model is implemented in an overall framework that allows for statistical variation of the structural, operational, and physico-chemical parameters such that a distribution of performance and durability is predicted based on the part-to-part variation in the manufacturing of the components. The model was applied to study the effect of the platinum loading, platinum/carbon ratio, and ionomer/catalyst ratio on the overall performance and durability. 


\section{Governing Equations}

The developed model is a 1-D approximation to a full MEA, Figure 1 shows the 1-D slice within a 2-D cross section and the indication of the agglomerate catalyst assumption used within the cathode catalyst layer.
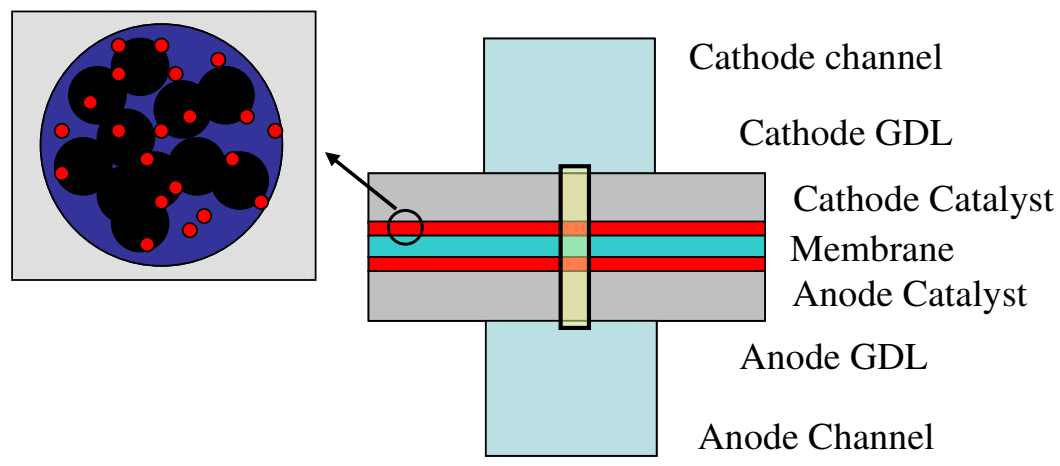

Figure 1: 1-D model domain within the 2-D cross section

The model accounts for the following physics:

- Charge Transport (Electrons and Protons)

- Ohm's Law

- Gas Transport $\left(\mathrm{H}_{2}, \mathrm{O}_{2}, \mathrm{~N}_{2}, \mathrm{H}_{2} \mathrm{O}\right)$

- Convection

- Diffusion (Mixture-based Fickian approach)

- Energy Transport

- Fourier's Law

- Ohmic Heating and Entropic Waste Heat

- Liquid Water Transport

- Saturation driven (with phase change)

- Platinum Dissolution, transport, and plating

- Potential driven dissolution/plating (two electron transfer)

The material compositions and layer properties are linked to the governing equations via the following flow diagram: 


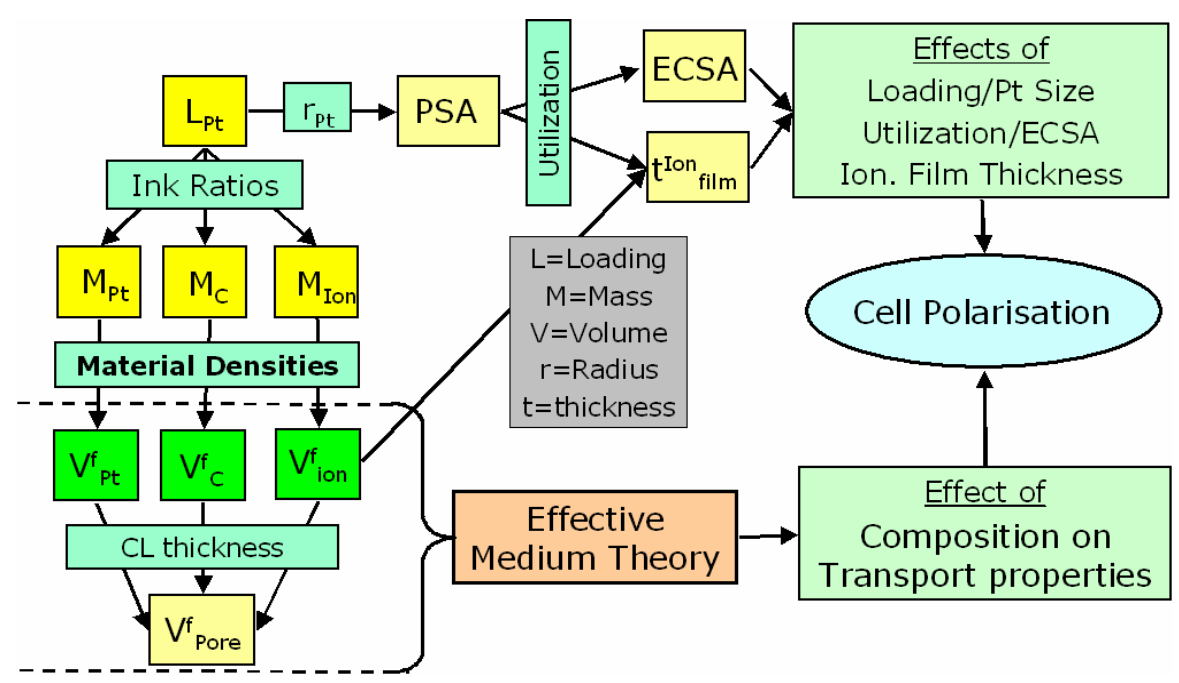

Figure 2: Catalyst Layer composition linkage to the governing equations

The statistical variation of the key input parameters was implemented by allowing for a specified statistical distribution of a given parameter and then performing a sampling operation of the parameter space at run time. Figure 3 shows an example of a random distribution taken around the platinum loading of the cathode catalyst layer.

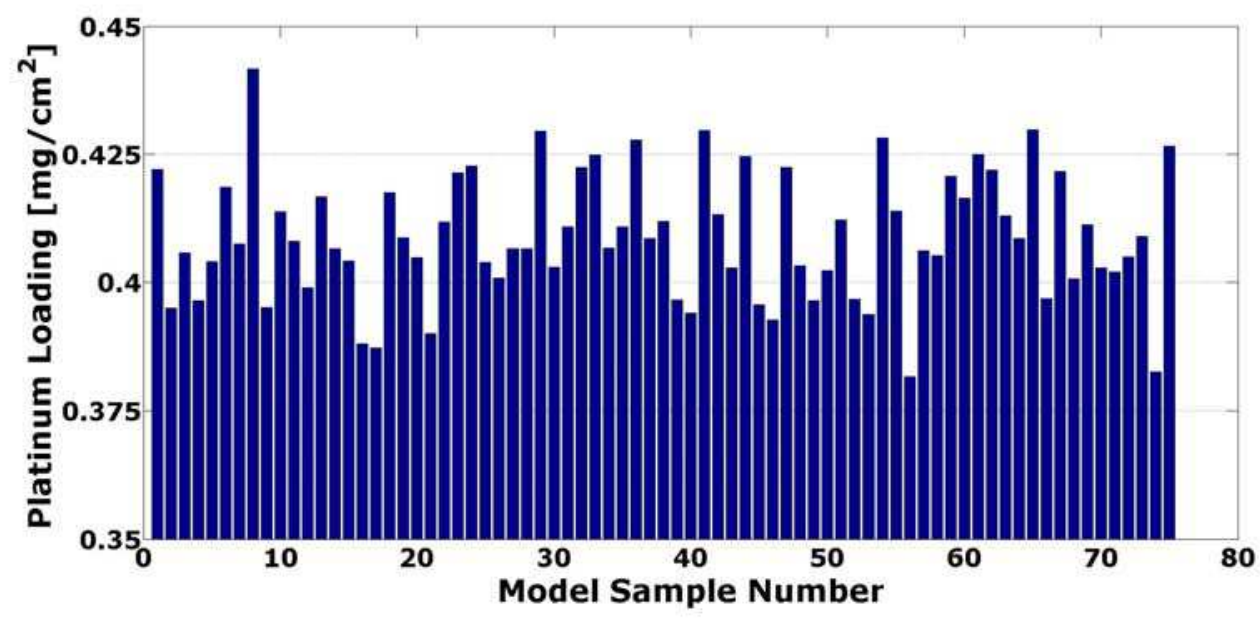

Figure 3: Statistical variation of platinum loading based on 75 successive model runs.

Overall, the statistical sampling was performed on both operational conditions and layer composition parameters; Table 1 shows a range of compositional and physico-chemical properties and their respective variations. 
Table 1: Statistical variation on Input Parameters

\begin{tabular}{|c|c|}
\hline Component Properties & \% Deviation (1 Std Dev) \\
\hline Catalyst/Catalyst Layer & $+/-8 \%$ \\
\hline Thickness (microns) & \\
\hline Weight Ratios (\%) & $+/-1 \%$ \\
\hline Pt:C & $+/-1 \%$ \\
\hline (Pt:C):Ionomer & $+/-5 \%$ \\
\hline Pt Loading [mg/cm^2] & $+/-10 \%$ \\
\hline Pt size & fixed \\
\hline Tafel Slope [mV/dec] & $+/-10 \%$ \\
\hline Jo [A/cm^2 pt] & fixed \\
\hline GDL & $+/-3 \%$ \\
\hline Porosity & $+/-5 \%$ \\
\hline Tortuosity & \\
\hline Thickness & $+/-2 \%$ \\
\hline Membrane & \\
\hline Thickness & \\
\hline
\end{tabular}

\section{Results and Discussion}

The model was used to simulate the performance and statistical variation in 20 baseline MEAs of similar composition. From Figure 4, it can clearly be seen that there exists a combination of part-to-part variations that result in an increased sensitivity and, subsequently, lower cell voltages at the higher current densities. Overall, the model shows the capability to capture both the average performance and distribution in the performance of the unit cell on a MEA-to-MEA basis, provided the variations of the MEA characteristics are known.

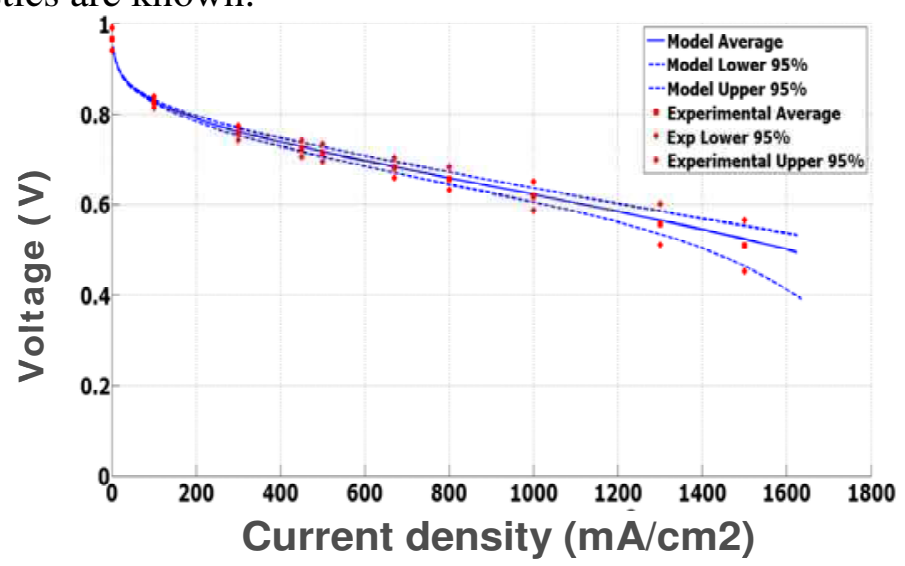

Figure 4 Validation of the Performance Model based on the statistical simulation of the baseline MEA.

Following the statistical validation using the baseline MEA, the model was used to study the effect of platinum loading reductions from $0.5-0.05 \mathrm{mg} / \mathrm{cm}^{2}$. In Figure 5 , the model shows the capability to capture the effect of the Pt loading reductions, in particular the ability to describe the changes in the reaction penetration into the catalyst layer due to changes in proton penetration, oxygen transport, and liquid water content. Further, in Figure 5, it is also seen that the model adequately captured the increased sensitivity to oxygen partial pressure seen at lower platinum loadings. The model results, shown in 
Figure 6, suggested that the cause of the increased sensitivity to lower oxygen partial pressures seen at the lower platinum loadings was, at least partially, due to higher liquid water saturation levels within the MEA, particularly within the electrode itself.
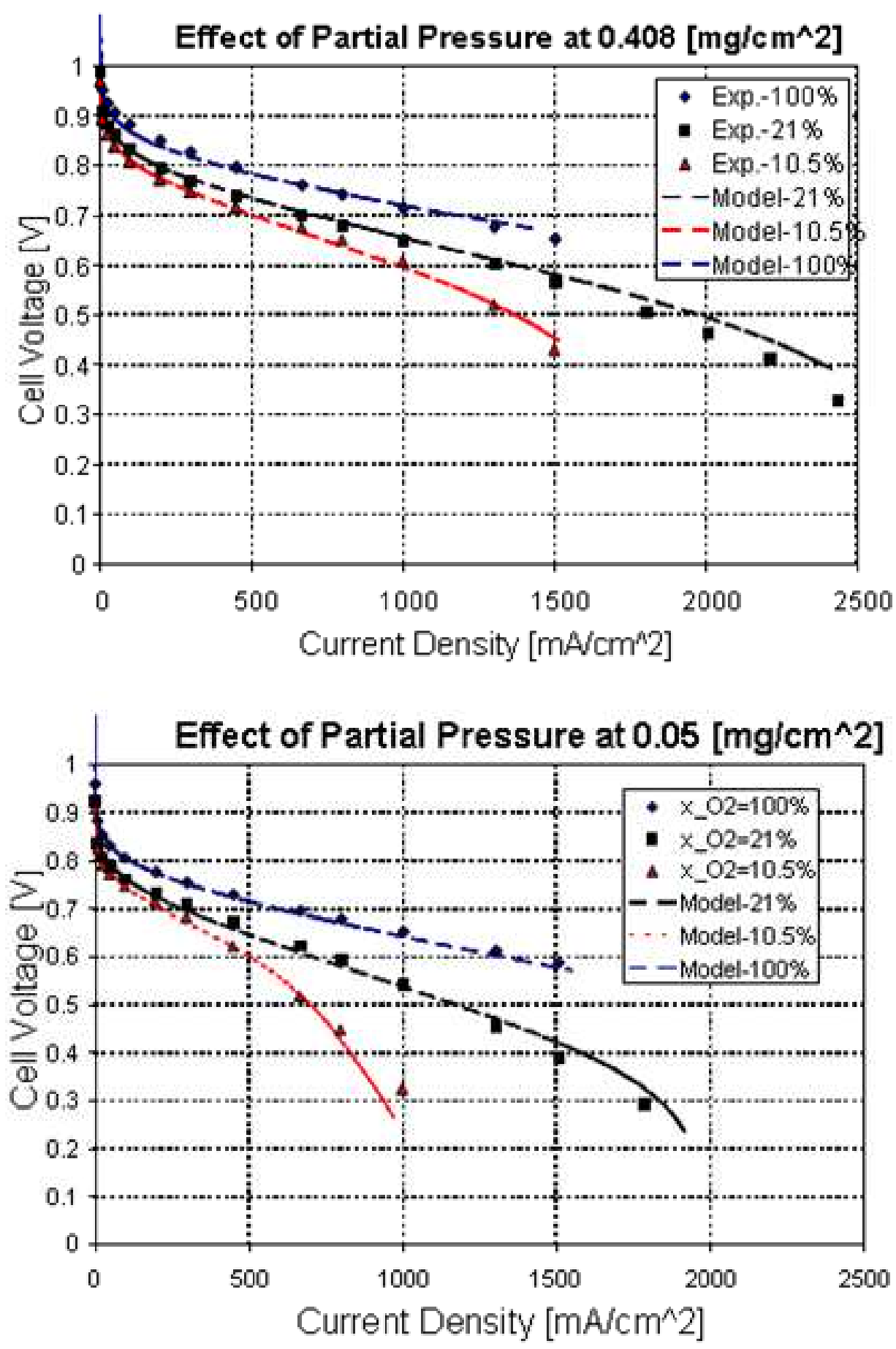

Figure 5: Validation of the MEA Performance Model for high and low Pt loadings at 3 different oxygen mole fractions. 

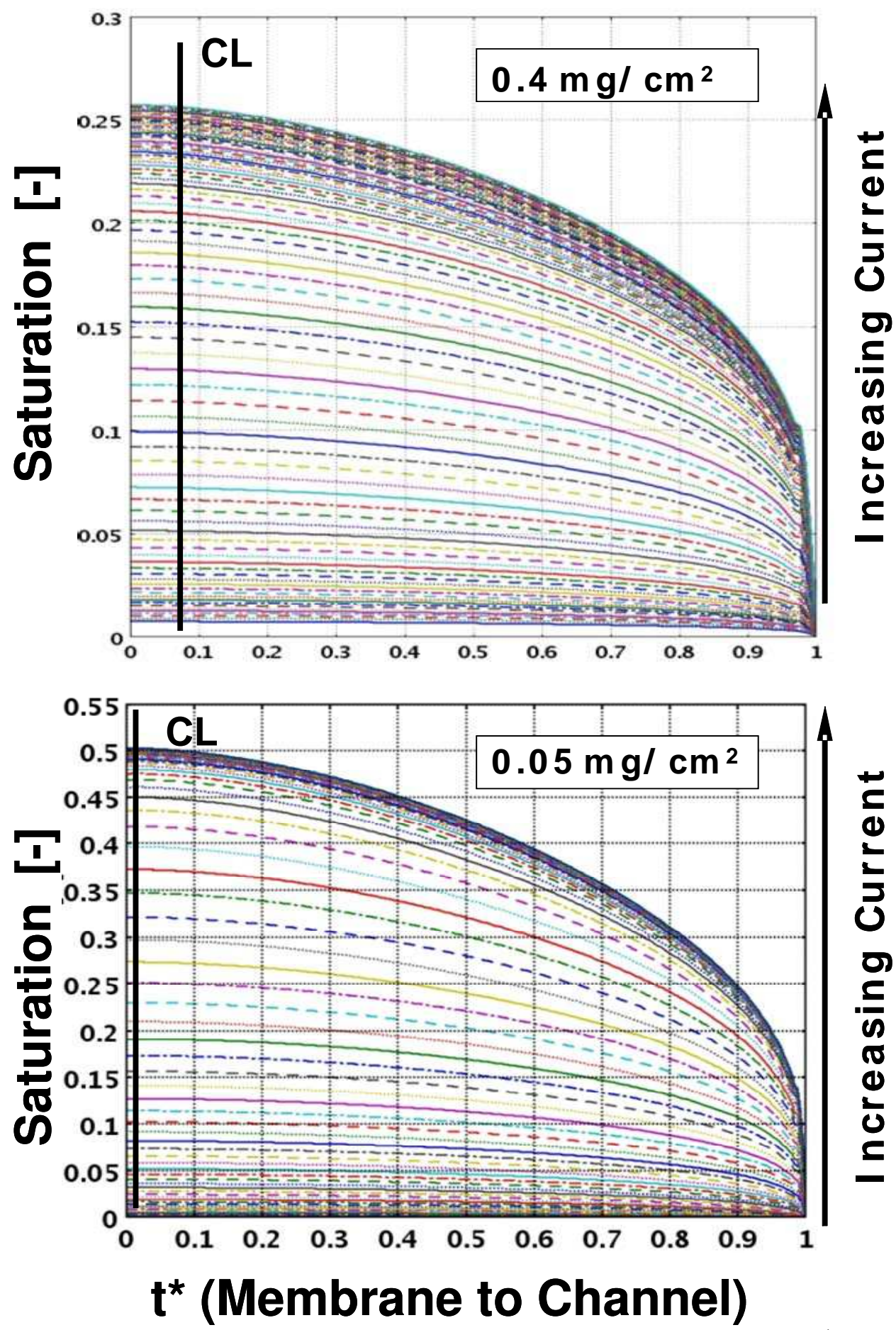

Figure 6: Water saturation as a function of non-dimensional distance $\left(\mathrm{t}^{*}\right)$ across the MEA.

The validated BOL model was then used to simulate the affect of Accelerated Stress Test (AST) protocols in which a series of different durability protocols were applied. These protocols ranged from potential holds, square waves, and triangle waves. Figure 7 shows an example of a triangle wave with a $1.1 \mathrm{~V}$ upper potential and the corresponding average platinum oxide coverage of the cathode catalyst. 


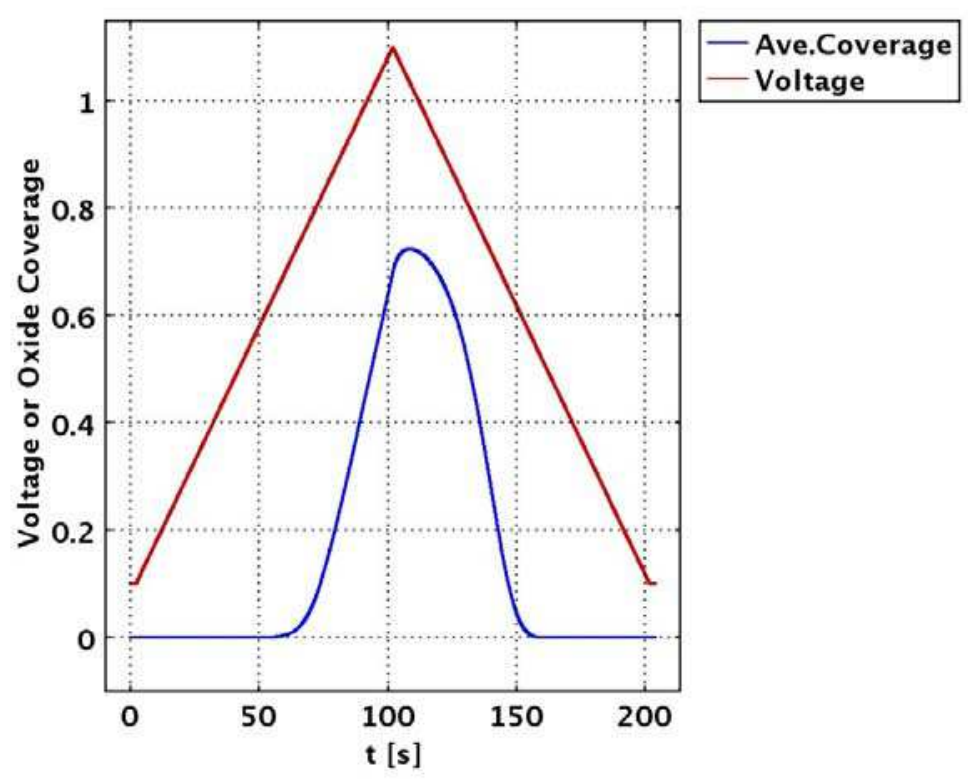

Figure 7: Triangle wave voltage profile and the cathode catalyst oxide coverage during sweep.

The model results from the AST cycling showed, consistent with experimental SEM imaging, that the platinum dissolution occurred preferentially in the region of the electrode directly adjacent the membrane resulting in changes in the reaction penetration of the oxygen reduction reaction within the layer. Further, this effect was found to be more influential in the lower loaded, thinner electrodes.

\section{Conclusions}

A statistical-based MEA performance model was developed and used to study the role of property variations. It was found that property variations played a role in the sensitivity to liquid water transport with certain combinations of parameters results in significantly reduced performance. The model was then applied to the study the effect of platinum loading and other compositional variations. It was found that the increase in liquid water in the liquid water within the electrode structure, at least partially, plays a role in the increased performance losses seen under lower platinum loadings. Finally, the model was applied to degradation in which a series of different cycling protocols were studied. It was found that the model predictions were similar to those of the experimental imaging in which platinum was seen to dissolve in the region adjacent to the membrane. This effect was observed to effect the reaction distribution in the catalyst layer, with a lengthening of the transport path for protons. 


\section{Acknowledgement}

The authors acknowledge financial assistance from U.S. Department of Energy DEEE0000466.

\section{References}

1. S. Wessel, "Development of Micro-Structural Mitigation Strategies for PEM Fuel Cells:

Morphological Simulations and Experimental Approaches", US DOE Merit Review FC049 (2012).

2. A.P. Young, J. Stumper, E. Gyenge, ESC, 156 (2009) B913-B922.

3. S. Arisetty, X. Wang, R.K. Ahluwalia, R. Mukundan, R. Borup, J. Davey, D. Langlois, F. Gambini, O. Polevaya, S. Blanchet, J. Electrochemical Society, 159 (5) B455-B462 (2012).

4. Y-H. Cho, J.W. Lim, Y.S. Kang,Y-H. Cho, O-H. Kim, N_H. Kwon, O. J. Kwon, WS. Yoon, H. Choe, Y_E. Saung, International J. of Hydrogen Energy, 37, 2490-2497 (2012). 